\title{
Weight gain and dietary intake during pregnancy in industrialized countries - a systematic review of observational studies
}

\author{
Ina Streuling', Andreas Beyerlein ${ }^{1} *$, Eva Rosenfeld ${ }^{2}$, \\ Britta Schukat ${ }^{1}$ and Rüdiger von Kries ${ }^{1}$ \\ ${ }^{1}$ Institute of Social Paediatrics and Adolescent Medicine, \\ Division of Epidemiology, Ludwig-Maximilians- \\ University Munich, Germany \\ ${ }^{2}$ Bavarian State Research Centre of Agriculture (LfL), \\ Institute of Food and Market, Munich, Germany
}

\begin{abstract}
Background: Gestational weight gain (GWG) above the recently recommended ranges is likely to be related to adverse pregnancy outcomes and therefore a challenge in industrialized countries.

Aims: We conducted a systematic review on observational studies in order to gain more evidence on whether diets with lower caloric/protein content or other diets might be associated with lower GWG.

Methods: We searched in MEDLINE and EMBASE for observational studies written in English or German reporting associations between diet and GWG in singleton pregnancies of healthy women in industrialized countries.

Results: We identified 12 studies which met the inclusion criteria. Five studies suggested significant positive associations between energy intake and GWG, whereas three found no significant association. Further significant positive associations of GWG were reported with respect to protein intake, animal lipids, energy density and a number of different food servings per day, whereas intake of carbohydrates and vegetarian diet were associated with less GWG.

Conclusions: We suggest that GWG might be reduced by lower energy intake in pregnancy.
\end{abstract}

Keywords: Energy intake; gestational weight gain; nutrition; pregnancy.

\footnotetext{
*Corresponding author:

Dr. Andreas Beyerlein, MSc

Ludwig-Maximilians University of Munich

Institute for Social Paediatrics and Adolescent Medicine

Division of Epidemiology

Heiglhofstr. 63

Munich 81377

Germany

Tel.: +49-89-71009-120

Fax: +49-89-71009-315

E-mail: andreas.beyerlein@med.uni-muenchen.de
}

\section{Introduction}

Gestational weight gain (GWG) results from complex physiological interactions between growth and development of the fetus and changes in maternal physiology and metabolism as well as in placental metabolism [19: 3-1]. Recently, the Institute of Medicine (IOM) published recommendations for GWG, which depend on maternal pre-pregnancy body mass index (BMI) [19: S2]. Gaining more weight than the recommended ranges (frequently called "excessive" GWG) may cause adverse short- and long-term pregnancy outcomes, such as maternal and offspring obesity, gestational diabetes mellitus, pregnancy-related hypertension, complications through labor and delivery and macrosomia $[6,10$, 14, 15, 17, 19: 5-1, 6-7, 20, 25, 26, 28, 30-32, 48, 49]. Therefore, GWG is of interest as a potentially modifiable prenatal risk factor. GWG, however, is determined by a number of factors which cannot be modified, such as maternal age, height, parity, duration of gestation or gender of the baby [19 (4), 47]. Interventions to modify pre-pregnancy BMI might be most promising but may be difficult because a large proportion of pregnancies are unplanned (in Germany, e.g., half of them [21]). General population interventions in young women have to be balanced against risks for eating disorders which are common in this age group [27, 41, 46]. A focus on potential dietary modifications has the advantage of better targeting.

GWG has increased in the last years in industrialized countries $[4,19: 2-1$ to $2-3,38]$ and it is a challenge to limit this trend. A number of interventional studies assessing the effects of energy or protein intake on perinatal outcomes including GWG has been summarized in a systematic review updated in 2009 [22].

The review concluded that GWG was significantly decreased by energy/protein restriction [22] based on only two studies $[8,9]$ comprising 253 women in total. The quality of both studies was poor due to inadequate allocation concealment, however. Furthermore, the studies were conducted in 1975 [9] and 1983 [8] and included only primiparous women from Scotland with either high GWG [9] or obesity [8], limiting the external validity of these findings.

Since the number of interventional studies addressing diet and GWG in the general population is limited [16], we performed a systematic review to assess whether observational studies reporting associations between maternal diet and GWG allow for further insight. We were specifically interested in whether diets with lower caloric/protein content or other diets might be associated with lower GWG. 


\section{Material and methods}

The databases MEDLINE (1950-2009) and EMBASE (1974-2009) were used to identify relevant articles on dietary determinants of GWG in healthy women. The systematic computerized literature search of published studies was carried out in November 2009 using the following search terms: ("weight gain pregnancy" OR "maternal weight gain" OR " gestational weight gain") AND ("nutrition", OR “diet" OR “energy intake" OR "protein intake" OR "dietary protein" OR "nutrition physiology"). After the search of the databases was finished, we searched manually the already identified articles and in the IOM guidelines [18, 19: S2] for further relevant articles.

We included only observational studies meeting the following criteria: written in English or German, dealing with healthy women having singleton pregnancies, published in population-based or hospital-based cohort studies in industrialized countries, without repeated pregnancies.

Not all studies explicitly mentioned whether they comprised only healthy women with singleton pregnancies; for example, only one study mentioned exclusion of women with gestational diabetes [23]. However, we assumed this if there was no indication (e.g., by adjustment) that women with severe diseases or multiple pregnancies were analyzed. We did not include studies which reported assessment of nutritional intake and GWG, but did not quantify associations between both.

\section{Results}

The literature search revealed $n=1917$ results. From these, 1722 studies were excluded because their study topic was different from our research question: most of the studies were excluded because either dietary intake or GWG was not recorded. One study [3] analyzed repeated pregnancies in the same mothers, i.e., 95 pregnancies in 54 women. As shown in Figure 1, 11 observational studies [1, 2, 5, 12, 23, 24, 33, $35,40,42,43,45]$ on the impact of dietary intake on GWG of healthy singleton pregnant women were finally identified as eligible for the systematic review. We detected one additional article [42] by a hand search.

Table 1 summarizes the setting of the 12 selected studies. Nine studies were performed in the USA and three in Europe. The sample sizes varied widely (50-2087 pregnant women). Two studies followed exclusively low-income pregnant teenagers [40, 43], whereas the other studies focused predominantly on adult pregnant women. Also, other inclusion and exclusion criteria of study participants, such as gestational age at entry or parity, varied considerably.

To assess nutritional intake, different dietary assessment measures were used (Table 2). Four studies assessed dietary habits by semi-quantitative food frequency questionnaires (FFQ), also asking for information on portion size [12, 23, $33,45]$, two used food records [5, 24], and five relied on recall methods [1, 2, 35, 40, 42]. One study used dietary records and recall methods [43]. Nutritional intake was assessed at least once or repeatedly at different points in time and assessed food intake over time periods of different length. Two studies, for example, assessed dietary habits during the last three months by an FFQ, implemented around the end of the second trimester [12] or in the first and in the third trimester [33]. With these instruments different nutritional aspects were considered. Some studies assessed intake of specific nutrients, e.g., protein, fat and carbohydrates or the intake of individual foods or food groups [23, 33, 40, 43]. Except for two studies [1, 42], food consumption was directly converted into some measure of energy intake. The study of Aaronson and Macnee [1] used a nutrition score to evaluate nutritional intake, in the study of Sloan et al. protein intake was recorded, which showed a significant correlation $(\mathrm{r}=0.81, \mathrm{P}<0.001)$ with caloric intake [42].

GWG was defined in different ways in the studies examined. The majority of studies [1, 2, 12, 24, 35, 43, 45] defined GWG as the difference between the maternal weight at her last visit to the prenatal care centre and (mostly self-

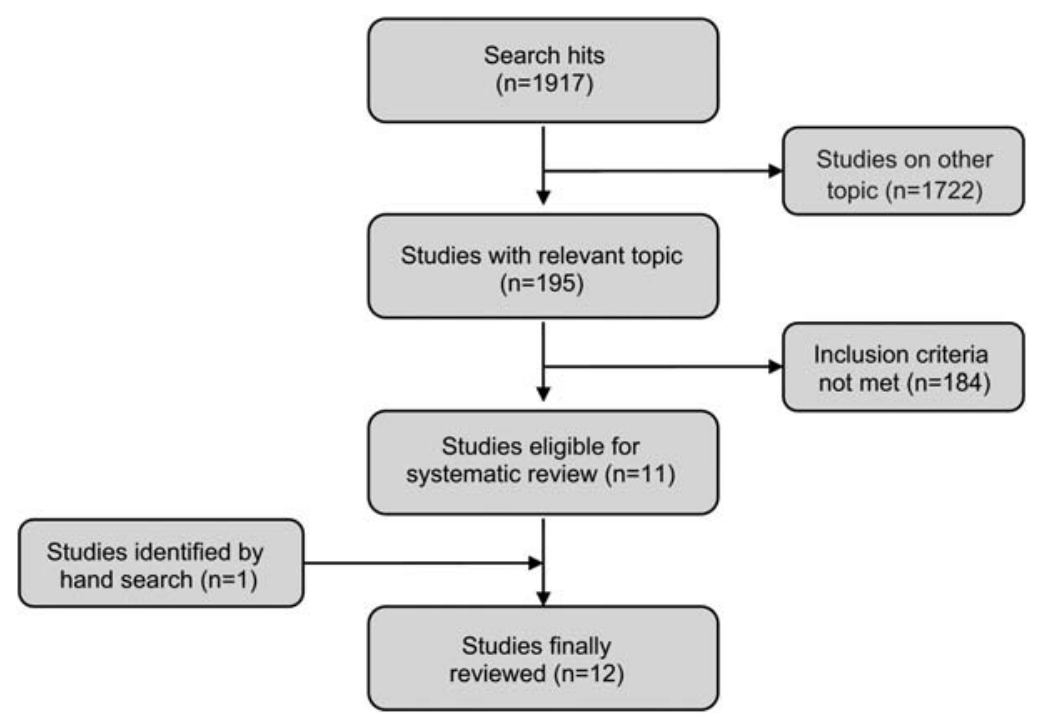

Figure 1 Identification and evaluation of the studies for the systematic review. 


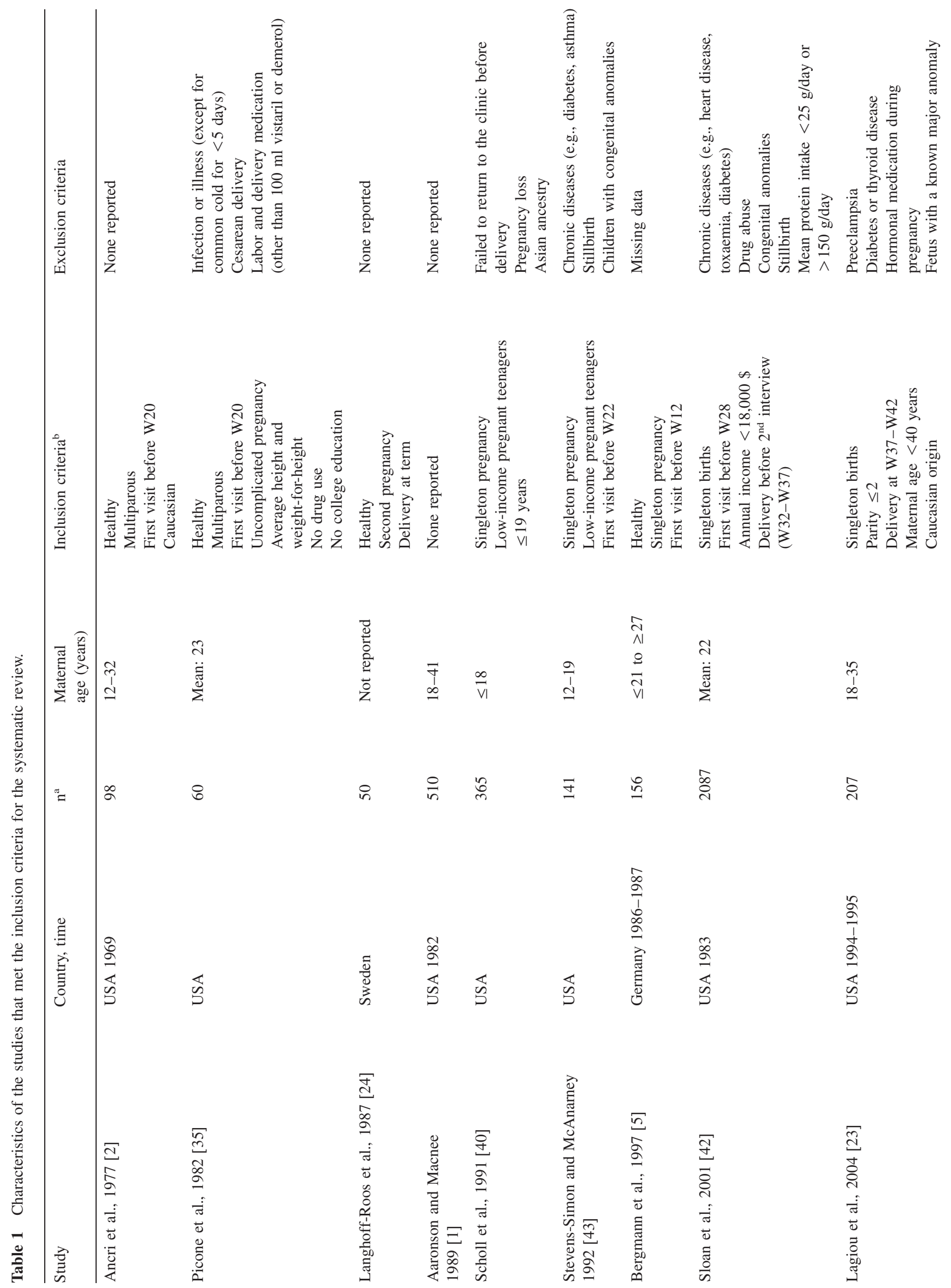


reported) pre-pregnancy weight. Others assessed GWG in different time frames during pregnancy [23, 33, 40]. Bergmann et al. [5] analyzed their data defining GWG as net GWG, subtracting offspring birth weight and weight of the placenta from weight gain between first and third trimester. No definition of GWG was given in the study of Sloan et al. [42].

Six studies [5, 12, 23, 24, 40, 45] adjusted for confounders, whereas the other six $[1,2,33,35,42,43]$ did not. Significantly $(\mathrm{P}<0.05)$ positive associations between energy intake and GWG were reported in five studies [5, 23, 33, 35, 40], three of them adjusted for confounders, whereas in three others (two adjusted) no significant association was found $[24,43,45]$. All three studies which assessed protein intake reported positive associations with GWG $[2,23,42]$ - in two of them (one with adjustment), these associations were significant $[2,23]$. Further significant associations of GWG were reported with respect to animal lipids [23], energy density [12], number of different food servings per day [1] - all of them associated with higher GWG; carbohydrates [23] and vegetarian diet [45] - both associated with lower GWG; with all but one [1] of these studies having adjusted for confounders.

\section{Discussion}

A systematic review applying a broad search strategy revealed 12 studies addressing the association between dietary intake in pregnancy and GWG. A number of studies partly adjusted for confounding factors-suggested positive associations between energy/protein intake and GWG.

These findings indicate that restrictions in energy intake during pregnancy might be a potential intervention strategy to avoid GWG above the IOM recommendations. Additionally, a diet with less intake of protein, but higher intake of carbohydrates, e.g., by reducing the amount of meat in the diet appeared to be associated with lower GWG although caution should be made to account for the well-known increased protein requirements in pregnancy $[13,34]$. Whereas the increased iron requirement during pregnancy can be met by iron supplementation, careful balancing is needed in order to meet the micronutrients requirements regarding vitamin B12 and possibly iodine and zinc [13]. The most effective intervention, however, might be a combination of a healthy diet and moderate physical activity as suggested by Stuebe et al. [45].

Unfortunately, the studies identified varied considerably with respect to dietary assessment and definition of GWG, so that we were unable to provide a summary effect estimate. We were also unable to assess publication bias, since conventional approaches like funnel plots require comparable measures for the strength of the association [7].

It is likely that the strength of the relationship between nutrition and GWG has been confounded by other factors if no adequate adjustment was performed. For example, in the study of Sloan et al. the amount of protein intake in pregnancy was negatively associated with maternal age, maternal 


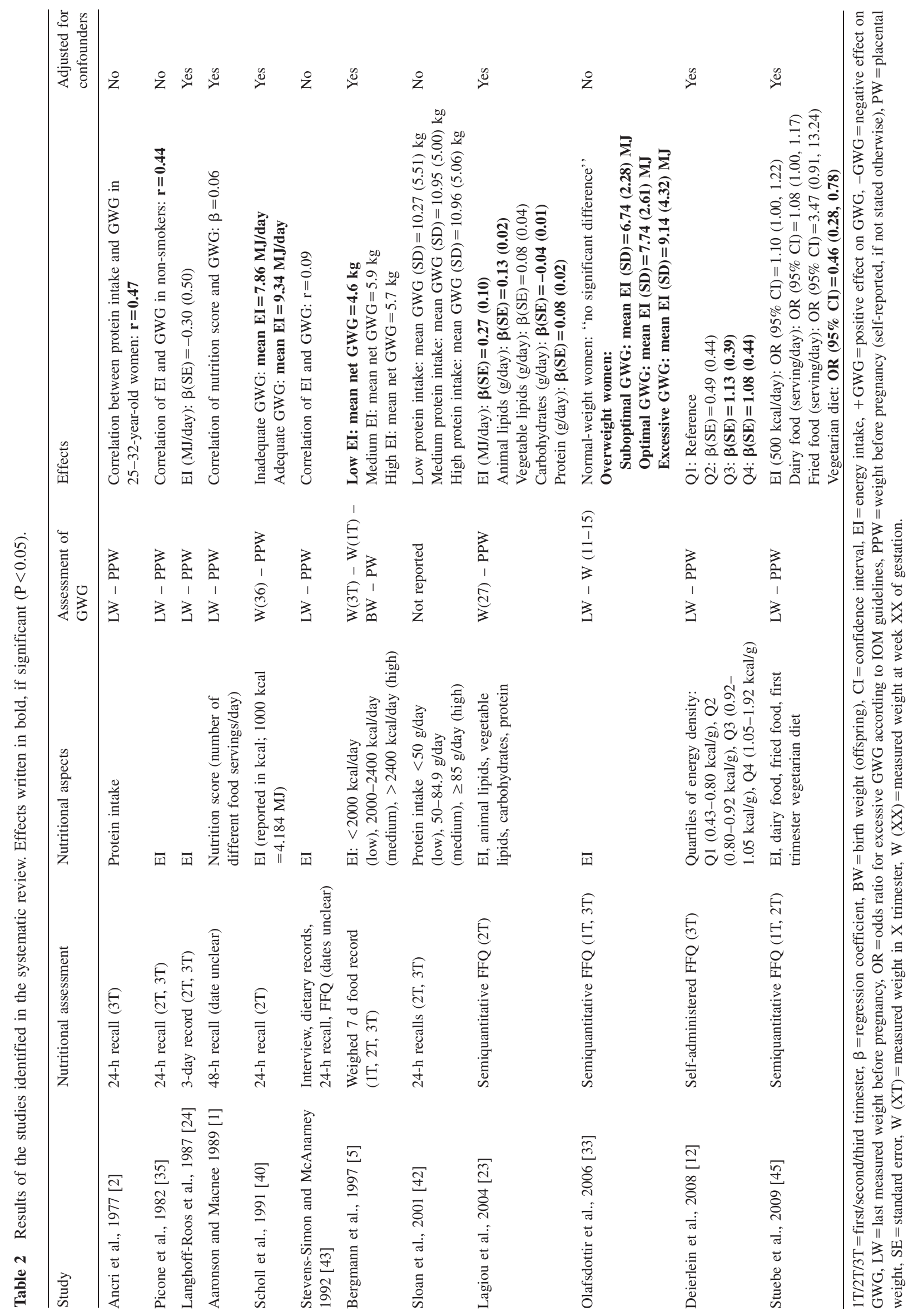


BMI and smoking status, but positively with GWG [42]. Maternal age is an important confounding factor. The significance of maternal growth of adolescents during pregnancy and its implications for GWG are still controversial [39, 44]. Additionally, given the trend towards delayed childbearing age, one should focus on GWG of pregnant women older than 35 years. Older women showed significantly lower mean GWG than younger women [36]. Pre-pregnancy BMI is known to be an important effect modifier of GWG: total GWG has been reported to be lower on average in women with high BMI $[11,28]$. However, since maternal BMI also has an impact on various short- and long-term outcomes, such as preeclampsia or offspring overweight, it seems necessary to perform analyses with stratification for maternal BMI $[6,10,28,30]$. There is also evidence that cigarette smoking is inversely associated with GWG [37]. Parity is also known to have a significant impact on GWG [6, 29]. Bergmann et al. [5] showed that multigravid women with high BMI gained less weight than primigravid women with a high BMI, whereas primigravid women with a high BMI gained much more weight than primi- and multigravid women with medium or low BMI.

Although the data identified in our systematic review of observational studies do not allow quantifying the potential effect of modifications of maternal diet in pregnancy on GWG, it appears to suggest that reducing energy intake might contribute to reducing GWG above the recommendations.

\section{Acknowledgements}

This study was supported by the project "Perinatal Prevention of Obesity Development" (PEPO). PEPO is part of the German obesity network funded by the German Federal Ministry of education and research (BMBF). None of the authors had a conflict of interest. The contributions of the authors were as follows: E.R. wrote the first and subsequent drafts of the manuscript. A.B. revised the manuscript and wrote the final draft. I.S. and B.S. were responsible for searching and reviewing the literature. R.v.K. contributed to conception and design of the study and to the first and final draft of the manuscript. Parts of this work arose from the PhD thesis of I.S. at the medical department of the University of Munich (in preparation).

\section{References}

[1] Aaronson LS, Macnee CL. The relationship between weight gain and nutrition in pregnancy. Nurs Res. 1989;38:223-7.

[2] Ancri G, Morse EH, Clarke RP. Comparison of the nutritional status of pregnant adolescents with adult pregnant women. III. Maternal protein and calorie intake and weight gain in relation to size of infant at birth. Am J Clin Nutr. 1977;30: 568-72.

[3] Beal VA. Nutritional studies during pregnancy. II. Dietary intake, maternal weight gain, and size of infant. J Am Diet Assoc. 1971;58:321-6.

[4] Bergmann KE, Bergmann RL, Ellert U, Dudenhausen JW. [Perinatal risk factors for long-term health. Results of the German Health Interview and Examination Survey for Chil- dren and Adolescents (KiGGS)]. Bundesgesundheitsblatt Gesundheitsforschung Gesundheitsschutz 2007;50:670-6.

[5] Bergmann MM, Flagg EW, Miracle-McMahill HL, Boeing H. Energy intake and net weight gain in pregnant women according to body mass index (BMI) status. Int J Obes. 1997;21: 1010-7.

[6] Beyerlein A, Schiessl B, Lack N, von Kries R. Optimal gestational weight gain ranges for the avoidance of adverse birth weight outcomes: a novel approach. Am J Clin Nutr. 2009;90: 1552-8.

[7] Borenstein M, Hedges L, Higgins J, Rothstein H. Introduction to Meta-Analysis. John Wiley \& Sons Ltd; 2009. p. 277-92.

[8] Campbell D. Dietary restriction in obesity and its effect on neonatal outcome. In: Campbell DM, Gillmer MDG, editors. Nutrition in pregnancy. Proceedings of 10th Study Group of RCOG. RCOG: London, 1983. p. 243-50.

[9] Campbell DM, MacGillivray I. The effect of a low calorie diet or a thiazide diuretic on the incidence of pre-eclampsia and on birth weight. Br J Obstet Gynaecol. 1975;82:572-7.

[10] Cedergren MI. Optimal gestational weight gain for body mass index categories. Obstet Gynecol. 2007;110:759-64.

[11] Chu SY, Callaghan WM, Bish CL, D'Angelo D. Gestational weight gain by body mass index among US women delivering live births, 2004-2005: fueling future obesity. Am J Obstet Gynecol. 2009;200:271.e1-7.

[12] Deierlein AL, Siega-Riz AM, Herring A. Dietary energy density but not glycemic load is associated with gestational weight gain. Am J Clin Nutr. 2008;88:693-9.

[13] Deutsche Gesellschaft für Ernährung, Referenzwerte für die Nährstoffzufuhr, ed. D.A.CH. Vol. 1. Auflage. 2000, Frankfurt/ Main: Umschau/ Braus.

[14] DeVader SR, Neeley HL, Myles TD, Leet TL. Evaluation of gestational weight gain guidelines for women with normal prepregnancy body mass index. Obstet Gynecol. 2007;110: $745-51$.

[15] Dietz P, Callaghan W, Sharma A. High pregnancy weight gain and risk of excessive fetal growth. Am J Obstet Gynecol. 2009;51.e1-6.

[16] Dodd JM, Grivell RM, Crowther CA, Robinson JS. Antenatal interventions for overweight or obese pregnant women: a systematic review of randomised trials. BJOG 2010;117: 1316-26.

[17] Gunderson EP, Abrams B, Selvin S. The relative importance of gestational gain and maternal characteristics associated with the risk of becoming overweight after pregnancy. Int $\mathbf{J}$ Obes Relat Metab Disord. 2000;24:1660-8.

[18] Institute of Medicine, Nutrition during Pregnancy: Part I Weight Gain, ed. IOM. Washington: National Academy Press; 1990. p. 10.

[19] Institute of Medicine, Weight gain during Pregnancy: Reexamining the Guidelines. Washington: The National Academy Press; 2009.

[20] Kiel DW, Dodson EA, Artal R, Boehmer TK, Leet TL. Gestational weight gain and pregnancy outcomes in obese women: how much is enough? Obstet Gynecol. 2007;110:752-8.

[21] Klusmann A, Heinrich B, Stöpler H, Gärtner J, Mayatepek E, Von Kries R. A decreasing rate of neural tube defects following the recommendations for periconceptional folic acid supplementation. Acta Paediatr. 2005;94:1538-42.

[22] Kramer M, Kakuma R. Energy and protein intake in pregnancy. Cochrane Database of Systematic Reviews 2003. Issue 4.

[23] Lagiou P, Tamimi RM, Mucci LA, Adami HO, Hsieh CC, Trichopoulos D. Diet during pregnancy in relation to maternal weight gain and birth size. Eur J Clin Nutr. 2004;58:231-7. 
[24] Langhoff-Roos J, Lindmark G, Kylberg E, Gebre-Medhin M. Energy intake and physical activity during pregnancy in relation to maternal fat accretion and infant birthweight. Br J Obstet Gynaecol. 1987;94:1178-85.

[25] Mamun AA, O'Callaghan M, Callaway L, Williams G, Najman J, Lawlor DA. Associations of gestational weight gain with offspring body mass index and blood pressure at 21 years of age: evidence from a birth cohort study. Circulation 2009;119:1720-7.

[26] Moreira P, Padez C, Mourão-Carvalhal I, Rosado V. Maternal weight gain during pregnancy and overweight in Portuguese children. Int J Obes. 2007;31:608-14.

[27] Nichols SD, Dookeran SS, Ragbir KK, Dalrymple N. Body image perception and the risk of unhealthy behaviours among university students. West Indian Med J. 2009;58:465-71.

[28] Nohr EA, Vaeth M, Baker JL, Sørensen TIA, Olsen J, Rasmussen KM. Combined associations of prepregnancy body mass index and gestational weight gain with the outcome of pregnancy. Am J Clin Nutr. 2008;87:1750-9.

[29] Nohr EA, Vaeth M, Baker JL, Sørensen TIA, Olsen J, Rasmussen KM. Pregnancy outcomes related to gestational weight gain in women defined by their body mass index, parity, height, and smoking status. Am J Clin Nutr. 2009;90: 1288-94.

[30] Oken E, Kleinman KP, Belfort MB, Hammitt JK, Gillman MW. Associations of gestational weight gain with short- and longer-term maternal and child health outcomes. Am J Epidemiol. 2009;170:173-80.

[31] Oken E, Rifas-Shiman SL, Field AE, Frazier AL, Gillman MW. Maternal gestational weight gain and offspring weight in adolescence. Obstet Gynecol. 2008;112:999-1006.

[32] Oken E, Taveras EM, Kleinman KP, Rich-Edwards JW, Gillman MW. Gestational weight gain and child adiposity at age 3 years. Am J Obstet Gynecol. 2007;196:322.e1-8.

[33] Olafsdottir AS, Skuladottir GV, Thorsdottir I, Hauksson A, Steingrimsdottir L. Maternal diet in early and late pregnancy in relation to weight gain. Int J Obes. 2006;30:492-9.

[34] Ortega RM. Dietary guidelines for pregnant women. Public Health Nutr. 2001;4:1343-6.

[35] Picone TA, Allen LH, Schramm MM, Olsen PN. Pregnancy outcome in North American women. I. Effects of diet, cigarette smoking, and psychological stress on maternal weight gain. Am J Clin Nutr. 1982;36:1205-13.

[36] Prysak M, Lorenz RP, Kisly A. Pregnancy outcome in nulliparous women 35 years and older. Obstet Gynecol. 1995;85: $65-70$.

[37] Rush D. Examination of the relationship between birthweight, cigarette smoking during pregnancy and maternal weight gain. J Obstet Gynaecol Br Commonw. 1974;81:746-52.
[38] Schiessl B, Beyerlein A, Lack N, von Kries R. Temporal trends in pregnancy weight gain and birth weight in Bavaria 2000-2007: slightly decreasing birth weight with increasing weight gain in pregnancy. J Perinat Med. 2009;37:374-9.

[39] Scholl TO, Hediger ML. A review of the epidemiology of nutrition and adolescent pregnancy: maternal growth during pregnancy and its effect on the fetus. J Am Coll Nutr. $1993 ; 12: 101-7$.

[40] Scholl TO, Hediger ML, Khoo CS, Healey MF, Rawson NL. Maternal weight gain, diet and infant birth weight: correlations during adolescent pregnancy. J Clin Epidemiol. 1991; 44:423-8.

[41] Sepulveda A, Carrobles JA, Gandarillas AM. Associated factors of unhealthy eating patterns among Spanish university students by gender. Span J Psychol. 2010;13:364-75.

[42] Sloan NL, Lederman SA, Leighton J, Himes JH, Rush D. The effect of prenatal dietary protein intake on birth weight. Nutr Res. 2001;21:129-39.

[43] Stevens-Simon C, McAnarney ER. Determinants of weight gain in pregnant adolescents. J Am Diet Assoc. 1992;92: 1348-51.

[44] Stevens-Simon C, McAnarney ER, Roghmann KJ. Adolescent gestational weight gain and birth weight. Pediatrics 1993;92:805-9.

[45] Stuebe AM, Oken E, Gillman MW. Associations of diet and physical activity during pregnancy with risk for excessive gestational weight gain. Am J Obstet Gynecol. 2009;201:58.e1-8.

[46] Vander Wal JS, Thelen MH. Eating and body image concerns among obese and average-weight children. Addict Behav. 2000;25:775-8.

[47] Viswanathan M, Maria Siega-Riz A, Moos M-K, Deierlein A, Mumford S, Knaack J, et al. Outcomes of maternal weight gain. Evid Rep Technol Assess (Full Rep), 2008:1-223.

[48] von Kries R, Ensenauer R, Beyerlein A, Amann-Gassner U, Hauner H, Rosario AS. Gestational weight gain and overweight in children: results from the cross-sectional German KiGGS study. Int J Pediatr Obes. 2010.

[49] Wrotniak BH, Shults J, Butts S, Stettler N. Gestational weight gain and risk of overweight in the offspring at age $7 \mathrm{y}$ in a multicenter, multiethnic cohort study. Am J Clin Nutr. 2008; 87:1818-24.

The authors stated that there are no conflicts of interest regarding the publication of this article.

Received May 12, 2010. Revised August 11, 2010. Accepted August 13, 2010. Previously published online November 11, 2010. 